\title{
Topological Electronic Structure and Its Temperature Evolution in Antiferromagnetic Topological Insulator $\mathrm{MnBi}_{2} \mathrm{Te}_{4}$
}

\author{
Y. J. Chen, ${ }^{1, *}$ L. X. Xu, ${ }^{2,3,4, *}$ J. H. Li, ${ }^{1, *}$ Y. W. Li, ${ }^{5, *}$ H. Y. Wang $\oplus^{2,4}$ C. F. Zhang, ${ }^{6}{ }^{6}$ H. Li, ${ }^{7,8}$ Y. Wu, ${ }^{1,8}$ A. J. Liang, ${ }^{2,9}$ \\ C. Chen, ${ }^{2,9}$ S. W. Jung $\odot,{ }^{10}$ C. Cacho, ${ }^{10}$ Y. H. Mao, ${ }^{6}$ S. Liu, ${ }^{2}$ M. X. Wang, ${ }^{2}$ Y. F. Guo, ${ }^{2}$ Y. Xu,${ }^{1,11,12}$ \\ Z. K. Liu, ${ }^{2, \$}$ L. X. Yang $®{ }^{1,11, \dagger}$ and Y. L. Chen ${ }^{1,2,5, \S}$ \\ ${ }^{1}$ State Key Laboratory of Low Dimensional Quantum Physics, Department of Physics, \\ Tsinghua University, Beijing 100084, China \\ ${ }^{2}$ School of Physical Science and Technology, ShanghaiTech University and CAS-Shanghai Science \\ Research Center, Shanghai 201210, China \\ ${ }^{3}$ Center for Excellence in Superconducting Electronics, State Key Laboratory of Functional Material \\ for Informatics, Shanghai Institute of Microsystem and Information Technology, \\ Chinese Academy of Sciences, Shanghai 200050, China \\ ${ }^{4}$ University of Chinese Academy of Sciences, Beijing 100049, China \\ ${ }^{5}$ Department of Physics, Clarendon Laboratory, University of Oxford, \\ Parks Road, Oxford OX1 3PU, United Kingdom \\ ${ }^{6}$ College of Advanced Interdisciplinary Studies, National University of Defense Technology, \\ Changsha 410073, China \\ ${ }^{7}$ School of Materials Science and Engineering, Tsinghua University, Beijing 10084, China \\ ${ }^{8}$ Department of Mechanical Engineering and Tsinghua-Foxconn Nanotechnology Research Center, \\ Tsinghua University, Beijing 100084, China \\ ${ }^{9}$ Advanced Light Source, Lawrence Berkeley National Laboratory, Berkeley, California 94720, USA \\ ${ }^{10}$ Diamond Light Source, Harwell Campus, Didcot OX11 ODE, United Kingdom \\ ${ }^{11}$ Frontier Science Center for Quantum Information, Beijing 100084, China \\ ${ }^{12}$ RIKEN Center for Emergent Matter Science (CEMS), Wako, Saitama 351-0198, Japan
}

(Received 16 July 2019; revised manuscript received 20 September 2019; published 21 November 2019)

The intrinsic magnetic topological insulator $\mathrm{MnBi}_{2} \mathrm{Te}_{4}$ exhibits rich topological effects such as quantum anomalous Hall effect and axion electrodynamics. Here, by combining the use of synchrotron and laser light sources, we carry out comprehensive and high-resolution angle-resolved photoemission spectroscopy studies on $\mathrm{MnBi}_{2} \mathrm{Te}_{4}$ and clearly identify its topological electronic structure. In contrast to theoretical predictions and previous studies, we observe topological surface states with diminished gap forming a characteristic Dirac cone. We argue that the topological surface states are mediated by multidomains of different magnetization orientations. In addition, the temperature evolution of the energy bands clearly reveals their interplay with the magnetic phase transition by showing interesting differences between the bulk and surface states, respectively. The investigation of the detailed electronic structure of $\mathrm{MnBi}_{2} \mathrm{Te}_{4}$ and its temperature evolution provides important insight into not only the exotic properties of $\mathrm{MnBi}_{2} \mathrm{Te}_{4}$, but also the generic understanding of the interplay between magnetism and topological electronic structure in magnetic topological quantum materials.

DOI: 10.1103/PhysRevX.9.041040

*These authors contributed equally to this work.

†xyang@tsinghua.edu.cn

*liuzhk@shanghaitech.edu.cn

§yulin.chen@physics.ox.ac.uk

Published by the American Physical Society under the terms of the Creative Commons Attribution 4.0 International license. Further distribution of this work must maintain attribution to the author(s) and the published article's title, journal citation, and DOI.
Subject Areas: Condensed Matter Physics,

Topological Insulators

\section{INTRODUCTION}

Topological quantum materials (TQMs) represent special classes of materials whose electronic structures can be characterized by topological invariants protected by certain symmetries, and the breaking of these symmetries can lead to intriguing topological phase transitions. Timereversal symmetry (TRS), for instance, is such an important symmetry in TQMs. While the TRS can protect the unique helical gapless surface states in topological insulators (TIs), it is broken in many exotic but interesting topological 
phases, such as quantum anomalous Hall (QAH) insulators [1-4], topological axion insulators [5-7], magnetic Dirac and Weyl semimetals [8-13], and compounds showing Majorana fermions [14]. However, despite the many theoretical proposals $[1,8-13,15]$, finding real TQMs with spontaneous broken TRS is challenging. For example, to date, the QAH effect could only be realized in TI $\left(\mathrm{Bi}_{1-x} \mathrm{Sb}_{x}\right)_{2} \mathrm{Te}_{3}$ thin films with fine-tuned magnetic doping (e.g., by $\mathrm{V}$ or $\mathrm{Cr}$ doping), under a temperature much lower than their Currie temperature [2,4]. Therefore, it is highly urged to search for stoichiometric TQMs with intrinsic magnetic structures for the realization of high-temperature QAH effect and other exotic topological properties.

Recently, it was realized that a layered compound, $\mathrm{MnBi}_{2} \mathrm{Te}_{4}$, could be a promising magnetic TI with intrinsic $A$-type antiferromagnetic (AFM) order [16-19] that can serve as an ideal platform for the realization of QAH effects and other interesting magnetic topological phases, including axion insulator and ideal Weyl semimetal phases [17]. Indeed, experimental efforts soon followed and topological phenomena such as the magnetic-field-induced QAH effect [20] and quantum phase transition from axion insulator to Chern insulator [21] were recently observed. In order to understand the rich properties and explore the full application potential of $\mathrm{MnBi}_{2} \mathrm{Te}_{4}$, both the surface and bulk electronic structures need to be experimentally verified.

Unfortunately, although there have been several attempts to resolve the band structures of this interesting compound, the results remain controversial and even conflicting [22-27]. First of all, the identification of the topological surface states (TSSs) remains elusive, leading to a significant difference in identifying the band gap of the surface states (due to the formation of magnetic order), ranging from $50 \mathrm{meV}$ [22] to a couple of hundred of meVs [23-26]. More intriguingly, these assumed gaps of TSSs persist well above the Néel temperature $\left(T_{N}\right)$ of $\mathrm{MnBi}_{2} \mathrm{Te}_{4}(25 \mathrm{~K}$, see Ref. [25]) and could even be observed at room temperature $[23,24]$. The order of magnitudes difference between the room temperature $(300 \mathrm{~K})$ and the $T_{N}$ of $\mathrm{MnBi}_{2} \mathrm{Te}_{4}(25 \mathrm{~K})$ makes it less convincing that the observed gap originates from the magnetic order. Finally, the fact that the measured gap size varies with photon energy [22-26] further alludes its bulk origin. These puzzles thus demand a systematic investigation on the electronic structure of $\mathrm{MnBi}_{2} \mathrm{Te}_{4}$ that can clearly distinguish the surface and bulk states in order to reveal their connection to the AFM transition-which can lay the foundation to the understanding of the interplay between the electronic structure, magnetic ordering, and the rich topological phenomena of this material.

In this work, combining the use of synchrotron and laser light sources, we carried out comprehensive and highresolution angle-resolved photoemission spectroscopy (ARPES) studies on $\mathrm{MnBi}_{2} \mathrm{Te}_{4}$ and clearly identified its topological electronic structure, including the characteristic TSSs. The broad photon energy range provided by the synchrotron light source allowed us to map out the subtle three-dimensional (3D) electronic structures, while the high-resolution laser light source made it possible to investigate the TSSs with great precision. Furthermore, by carrying out temperature-dependent measurements, we were able to observe the temperature evolution from both the bulk and surface-states bands, which shows interesting differences between their interplay with the magnetic phase transition: while the bulk states show clear splitting commencing at $T_{N}$, the gap of TSSs stays negligible within our energy resolution [about $2.5 \mathrm{meV}$ at temperature (7.5 K) well below $T_{N}$ ], which could be due to the highly delocalized nature of TSSs mediated by surface magnetic domains of different magnetization orientations. The identification of the detailed electronic structure of $\mathrm{MnBi}_{2} \mathrm{Te}_{4}$ will help us to understand its exotic properties. Our results also suggest that the magnetic and topological properties of $\mathrm{MnBi}_{2} \mathrm{Te}_{4}$ can be manipulated by aligning the magnetization, which sheds light on the design and realization of novel phenomena and applications. For example, by interfacing with van der Waals ferromagnets such as $\mathrm{Fe}_{3} \mathrm{GeTe}_{2}$ and $\mathrm{CrI}_{3}$ [28], it is possible to manipulate the topological electronic structure and properties of $\mathrm{MnBi}_{2} \mathrm{Te}_{4}$ and realize topological phase transition by tuning its magnetic states.

\section{METHODS}

\section{A. Crystal growth}

The single crystal of $\mathrm{MnBi}_{2} \mathrm{Te}_{4}$ was grown by a direct reaction of a stoichiometric mixture of $\mathrm{Bi}_{2} \mathrm{Te}_{3}$ and $\mathrm{MnTe}$, which were synthesized by reacting high-purity $\mathrm{Bi}$ (99.99\%, Adamas) and Te (99.999\%, Aladdin), and Mn (99.95\%, Alfa Aesar) and Te (99.999\%, Aladdin), respectively. The growth of high-quality $\mathrm{MnBi}_{2} \mathrm{Te}_{4}$ single crystals was carried out in a sealed silica ampoule under a dynamic vacuum. The ampoule was first heated to $973 \mathrm{~K}$. After being slowly cooled down to $864 \mathrm{~K}$, the crystal growth occurred during the long-term annealing at the same temperature and afforded millimeter-sized shiny single crystals of $\mathrm{MnBi}_{2} \mathrm{Te}_{4}$. The crystals were examined on a PANalytical Empyrean diffractometer with $\mathrm{Cu} K \alpha$ radiation to establish the correct phase and high quality of single crystal samples for the experiment. More details about sample growth and characterization including the stoichiometry are described elsewhere [29].

\section{B. Angle-resolved photoemission spectroscopy}

ARPES measurements were performed at beam line I05 of the Diamond Light Source (DLS), UK, beam line BL13U of National Synchrotron Radiation Laboratory (NSRL), China, beam line BL03U of Shanghai Synchrotron Radiation Facility (SSRF), China, and beam line 10.0.1 of the Advanced Light Source (ALS). The samples were cleaved in situ and measured under ultrahigh 
vacuum below $1 \times 10^{-10}$ Torr at DLS, $6 \times 10^{-11}$ Torr at NSRL, $6 \times 10^{-11}$ Torr at SSRF, and $3 \times 10^{-11}$ Torr at ALS. Data were collected by Scienta R4000, DA30 L, DA30 L, and R4000 analyzers at DLS, NSRL, SSRF, and ALS, respectively. The total convolved energy and angle resolutions were $15 \mathrm{meV}$ and $0.2^{\circ}$, respectively.

High-resolution laser-based ARPES measurements were performed on home-built setups $(h \nu=6.994 \mathrm{eV})$ at Tsinghua University and ShanghaiTech University. The samples were cleaved in situ and measured under ultrahigh vacuum below $6 \times 10^{-11}$ Torr. Data were collected by a DA30 L analyzer. The total convolved energy and angle resolutions were $2.5 \mathrm{meV}$ and $0.2^{\circ}$, respectively.

\section{First-principles calculations}

First-principles calculations were performed by density functional theory (DFT) using the Vienna ab initio simulation package. The plane-wave basis with an energy cutoff of $350 \mathrm{eV}$ was adopted. The electron-ion interactions were modeled by the projector augmented wave potential and the exchange-correlation functional was approximated by the Perdew-Burke-Ernzerhof-type generalized gradient approximation (GGA) [30]. The GGA $+U$ method was applied to describe the localized $3 d$ orbitals of $\mathrm{Mn}$ atoms, for which $U=4.0 \mathrm{eV}$ was selected according to our previous tests [16]. The structural relaxation for optimized lattice constants and atomic positions was performed with a force criterion of $0.01 \mathrm{eV} / \AA$ and by using the DFT-D3 method to include van der Waals corrections. Spin-orbit coupling was included in self-consistent calculations and the Monkhorst-Pack $k$-point mesh of $9 \times 9 \times 3$ was adopted. Surface state calculations were performed with the WANNIERTOOLS package [31], based on the tight-binding Hamiltonians constructed from maximally localized Wannier functions (MLWF) [32].

\section{RESULTS}

\section{A. Bulk band structure}

$\mathrm{MnBi}_{2} \mathrm{Te}_{4}$ crystallizes into a rhombohedral lattice with space group of $R \overline{3} m$ [26,33,34]. It exhibits a layered structure by staking van der Waals septuple Te-Bi-Te-MnTe-Bi-Te layers, which has an extra Mn-Te layer sandwiched at the middle of the well-known quintuple layer of $\mathrm{Bi}_{2} \mathrm{Te}_{3}$, as shown in Fig. 1(a). The high quality of the single crystalline samples used in this work is demonstrated by the single crystal x-ray diffraction patterns [Fig. 1(b)] and angle scan [Fig. 1(c)]. The magnetic susceptibility and electric transport measurements [Fig. 1(d)] clearly show the AFM phase transition at $T_{N}=25 \mathrm{~K}$. For the ARPES measurements, the sample was cleaved in situ with flat surfaces, showing clear layered structure [Fig. 1(b)]. Scanning tunneling microscopy measurement reveals a step height of about $1.37 \mathrm{~nm}$ on the sample surface, in good agreement with the thickness of the septuple layer $(1 / 3 c)$ in $\mathrm{MnBi}_{2} \mathrm{Te}_{4}$. The characteristic $\mathrm{Mn}, \mathrm{Bi}$, and $\mathrm{Te}$ core level peaks in $\mathrm{x}$-ray photoemission spectroscopy measurement further confirm the sample phase [Fig. 1(f)]. The broad band-structure mapping at the surface Dirac point $[\sim 0.27 \mathrm{eV}$ below the (a)

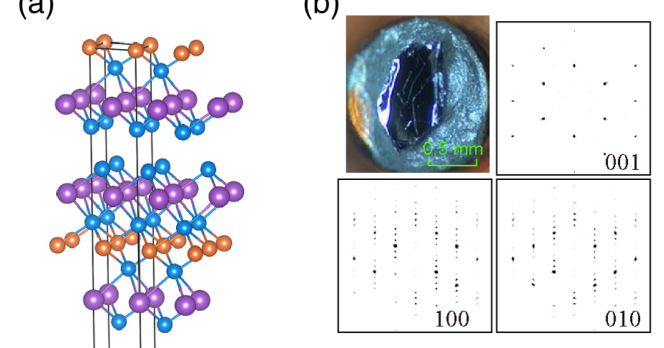

(e)

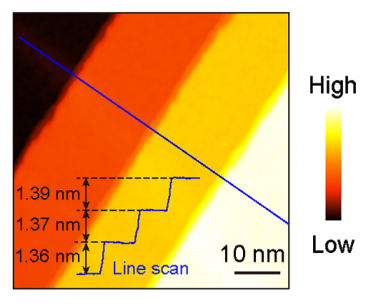

(c)

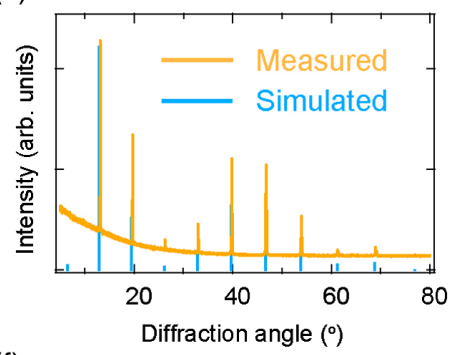

(f)

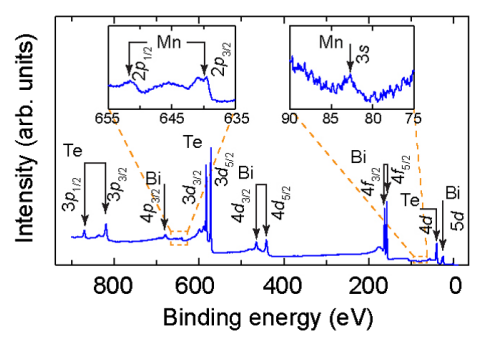

(d)

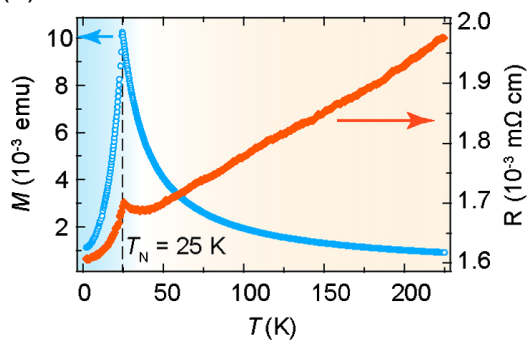

(g)

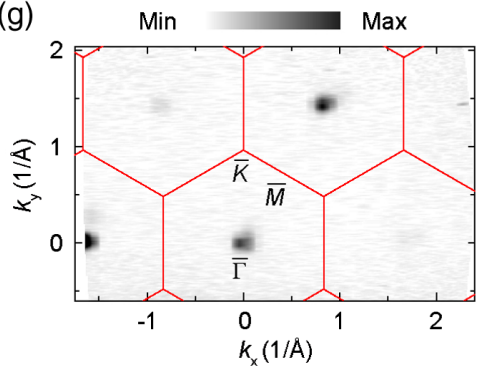

FIG. 1. Basic properties and characterization of $\mathrm{MnBi}_{2} \mathrm{Te}_{4}$ single crystals. (a) Schematic of the layered crystal structure of $\mathrm{MnBi}_{2} \mathrm{Te}_{4}$. (b) Image of $\mathrm{MnBi}_{2} \mathrm{Te}_{4}$ single crystal after cleavage and $\mathrm{x}$-ray diffraction patterns along different directions. (c) Angle scan of $\mathrm{x}$-ray diffraction along $c$ axis. (d) Magnetization (with magnetic field applied along $c$ axis) and resistance as functions of temperature. (e) Scanning tunneling microscopy mapping of surface topography obtained with $I=250 \mathrm{~mA}$ and $V=1 \mathrm{~V}$. (f) X-ray photoemission spectroscopy showing characteristic $\mathrm{Mn}, \mathrm{Bi}$, and Te core levels. (g) Constant energy contour near $270 \mathrm{meV}$ below the Fermi energy over multiple BZ; data were collected under $100 \mathrm{eV}$ photon energy at $18 \mathrm{~K}$. 

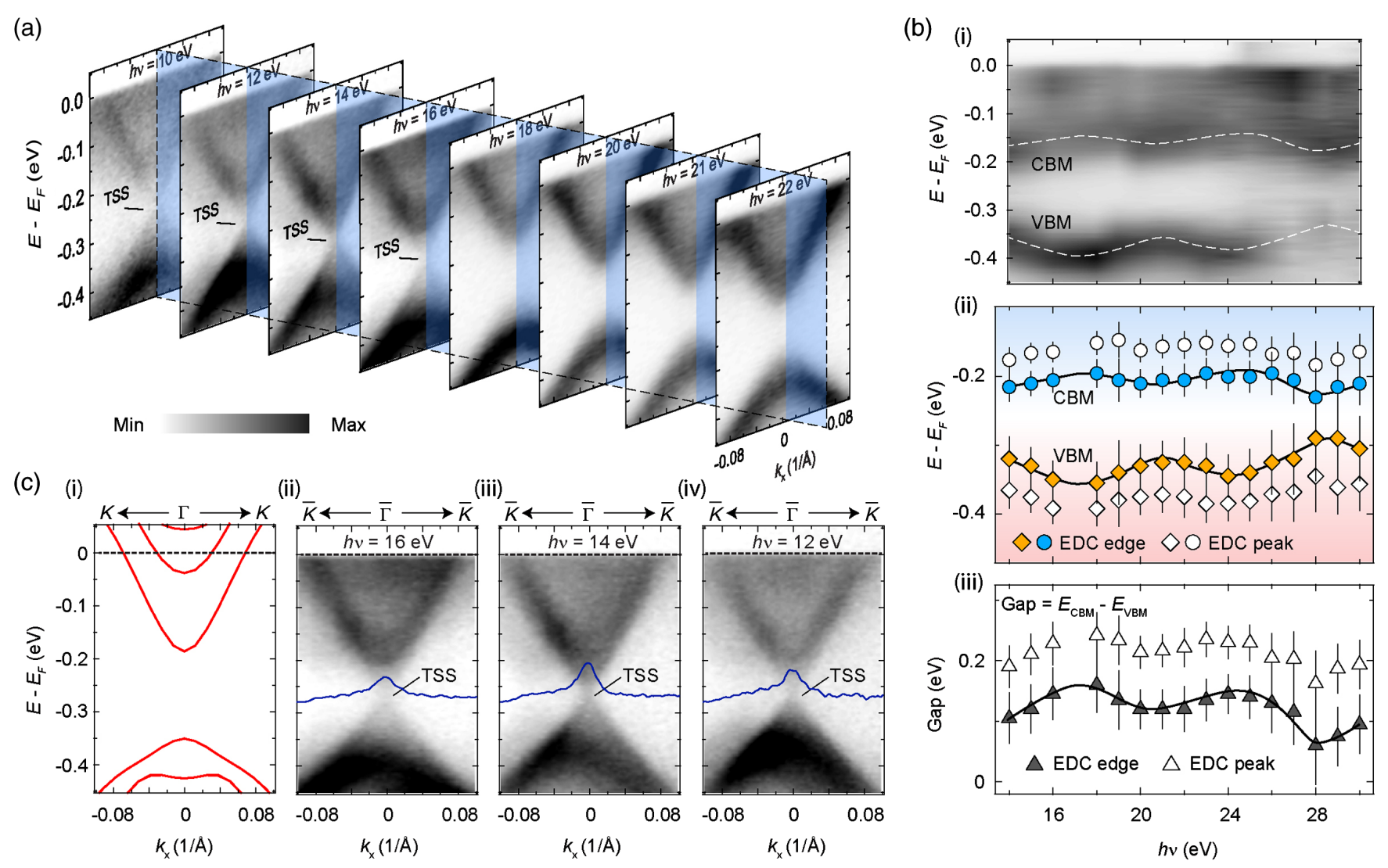

FIG. 2. Photon-energy-dependent band structure measurements on $\mathrm{MnBi}_{2} \mathrm{Te}_{4}$. (a) Band dispersion near $\bar{\Gamma}$ measured with different photon energies. The topological surface state (TSS) as marked is observed at photon energies lower than $16 \mathrm{eV}$. (b) Photon energy dependence of (i) energy distribution curve (EDC) near $\bar{\Gamma}$, (ii) extracted conduction band minimum (CBM) and valance band maximum (VBM), and (iii) bulk band gap. (c)(i) Calculated bulk band dispersion along $\Gamma K\left(k_{z}=0\right.$ plane). (c)(ii)-(c)(iv) Band dispersion obtained at low-photon energies showing characteristic TSSs. The insets are the momentum distribution curves (MDCs) integrated near the Dirac point. Data were collected at $14 \mathrm{~K}$.

Fermi energy $\left(E_{F}\right)$, as we discuss later] over multiple Brillouin zones (BZs) shows clear pointlike features with hexagonal symmetry [Fig. 1(g)], in agreement with the crystal structures of $\mathrm{MnBi}_{2} \mathrm{Te}_{4}$.

The bulk band structure of $\mathrm{MnBi}_{2} \mathrm{Te}_{4}$ is illustrated in Fig. 2. The conduction and valance bands form an inverted bulk gap $[16,17]$ whose magnitude varies with the photon energies used for the ARPES measurements [Figs. 2(a) and 2(b)], indicting its bulk nature. Depending on the methods used for gap extraction [35], the bulk gap size at different $k_{z}$ momentum varies from 180 to $220 \mathrm{meV}$ [fitted peak-peak gap in energy distribution curves (EDCs)] or 80 to $160 \mathrm{meV}$ (EDC leading edge gap), as summarized in Fig. 2(b)(iii). Interestingly, besides the bulk bands with very strong photoemission spectra intensity that agree well with the calculations [Fig. 2(c)(i)], one can notice that there is also weak spectra intensity within the bulk band gap [Figs. 2(c)(ii)-2(c)(iv)], showing an X-shaped dispersion centered at the $\bar{\Gamma}$ point, similar to the dispersion of the TSSs of other 3D TIs $[36,37]$. The TSSs could only be observed at photon energies below $18 \mathrm{eV}$, which may be due to matrix element and cross-section effects.

\section{B. Topological surface states}

To further investigate the TSSs in the bulk band gap, we carried out laser-based ARPES measurements with superb energy and momentum resolutions, as illustrated in Fig. 3. The evolution of band structures with different binding energy [Figs. 3(a) and 3(b)] clearly shows the TSSs with conical shape dispersion across the Dirac point located at $\sim 0.27 \mathrm{eV}$ below $E_{F}$ within the bulk band gap. The linear dispersion of TSSs can be directly seen in Fig. 3(c), with vanishing energy gap unresolved under the energy resolution of the experiments $(\Delta E \sim 2.5 \mathrm{meV})$, which can be better seen in the enlarged plot around the Dirac point [Fig. 3(d)].

Besides the TSSs, the high resolution in the measurement of laser-based ARPES also allows us to investigate the bulk states with great detail. From the spectra intensity map [Fig. 3(c)], the second derivative plot [Fig. 3(e)], or the stacking momentum distribution curves (MDCs) plot [Fig. 3(f)], one can clearly see three conduction bands. Interestingly, by comparing with theoretical calculation of bulk bands in Fig. 2(c)(i), we notice that an extra bulk band 


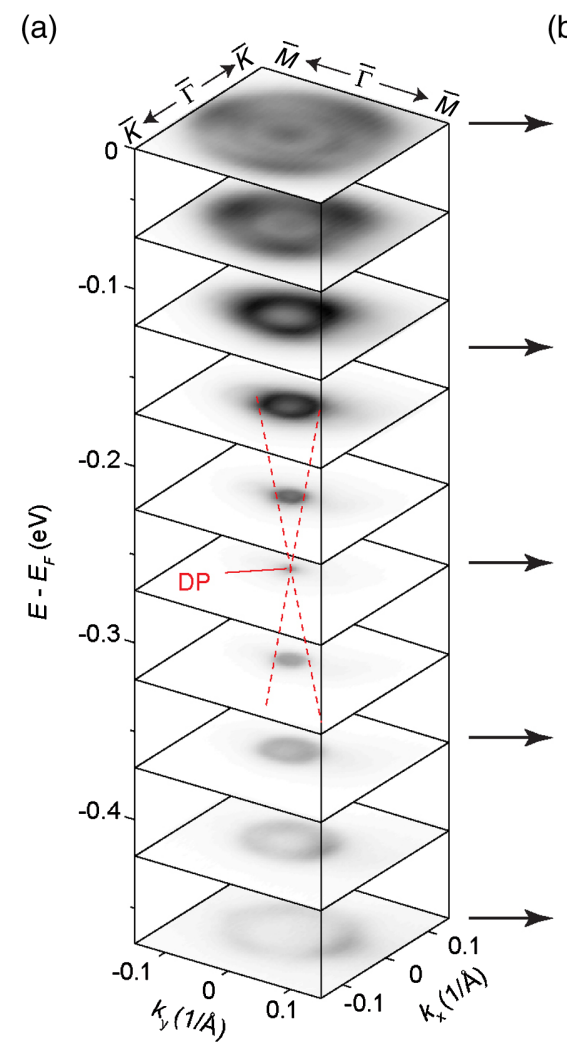

(b)

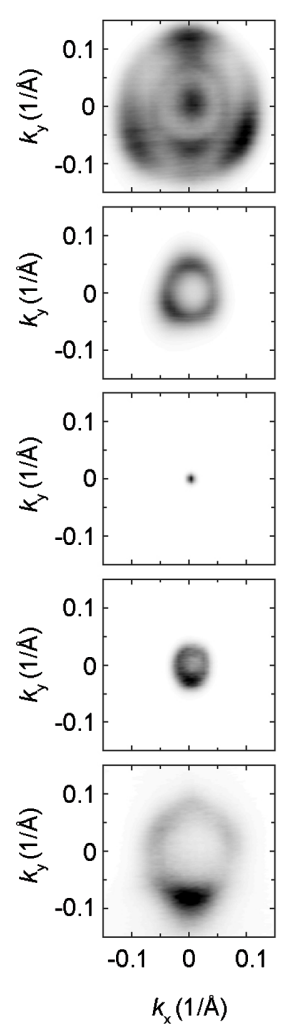

(c)

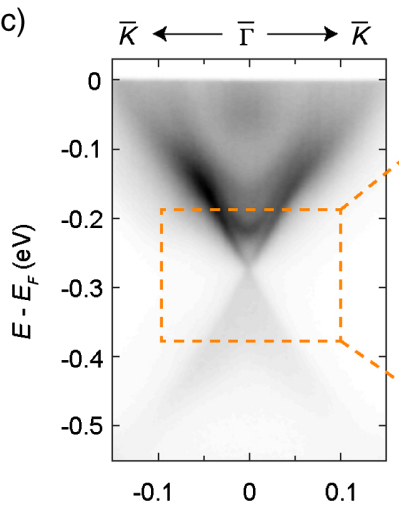

(e)

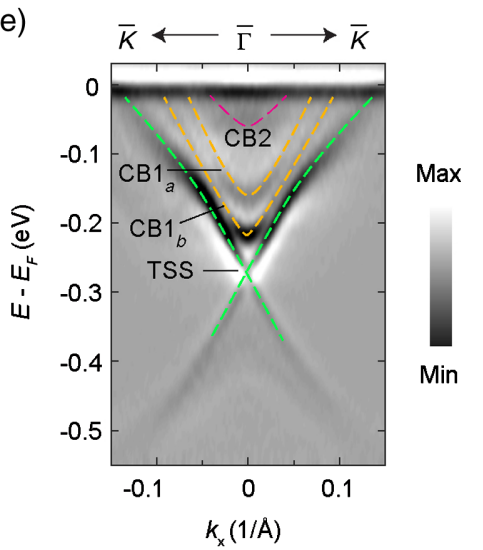

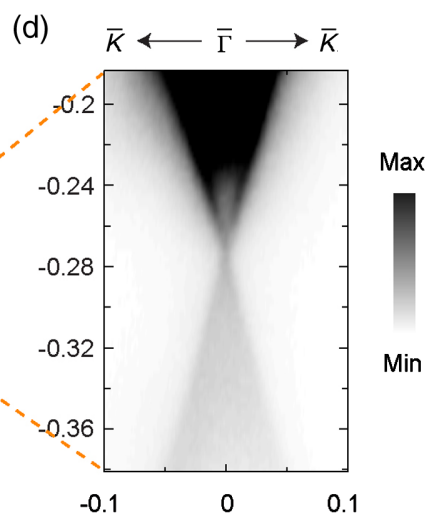

(f)

FIG. 3. Electronic structure of $\mathrm{MnBi}_{2} \mathrm{Te}_{4}$ measured with $6.994 \mathrm{eV}$ laser. (a),(b) Constant energy contours at different binding energies. (c) Band dispersion along the $\bar{\Gamma} \bar{K}$ direction with a clear Dirac cone formed by TSSs. (d) Enlarged plot of the dispersion near the Dirac point (DP). (e) Second derivative of ARPES intensity map in (c). The dashed curves are guides to eye for band dispersions. (f) Stacking plot of MDCs. The bulk conduction bands and TSSs with diminished gap are clearly observed. Data were collected at $7.5 \mathrm{~K}$.

is observed, whose origin is discussed later. We mark the observed conduction bands as $\mathrm{CB} 2, \mathrm{CB} 1_{a}$, and $\mathrm{CB} 1_{b}$, respectively, as shown in Figs. 3(e) and 3(f) (the reason for the naming is discussed below).

\section{Temperature evolution of the electronic structure of $\mathrm{MnBi}_{2} \mathrm{Te}_{4}$}

To understand the interplay between the band structure and the magnetic properties of $\mathrm{MnBi}_{2} \mathrm{Te}_{4}$, we carried out a series of temperature-dependent ARPES measurements across $T_{N}$, which are summarized in Fig. 4. For the bulk conduction bands, upon the increase of temperature, the $\mathrm{CB} 1_{a}$ and $\mathrm{CB}_{b}$ bands move toward each other and eventually merge into one single band (CB1) above $T_{N}$, as illustrated in Figs. 4(a) and 4(b). When the temperature is sequentially cooled down to below $T_{N}, \mathrm{CB} 1$ splits into $\mathrm{CB} 1_{a}$ and $\mathrm{CB}_{b}$ again, as can be clearly seen in Figs. 4(a)(vi) and 4(b)(vi); the same behavior has also been observed in the measurements on multiple samples and under various photon energies [35]. The side-by-side comparison between the band structure above and below $T_{N}$ can be seen in Fig. 4(c) for clarity, and the temperature evolution of the EDCs at the $\bar{\Gamma}$ point is summarized in Fig. 4(d), with the fitted peak-to-peak splitting of the $\mathrm{CB} 1_{a}$ and $\mathrm{CB} 1_{b}$ on multiple temperatures plotted in Fig. 4(e). The coincidence of the splitting temperature and the $T_{N}$ near $25 \mathrm{~K}$ indicates a correlation between the band splitting and the formation of the magnetic order. Although the observed bulk band splitting is beyond the expectation of an AFM system, it is accessible considering the surface ferromagnetic ordering, which has been shown to induce considerable exchange splitting in AFM EuRh${ }_{2} \mathrm{Si}_{2}$ [38]. On the other hand, according to first-principles calculations, $\mathrm{CB} 1_{a}$ and $\mathrm{CB} 1_{b}$ are mainly contributed by the Te $p_{z}$ orbital. When the temperature increases above $T_{N}$, the exchange-induced splitting vanishes, which has complicated influence on interlayer coupling and band dispersion, leading to the merging of the $\mathrm{CB} 1_{a}$ and $\mathrm{CB} 1_{b}$ bands.

\section{DISSCUSSION}

Interestingly, in contrast to the bulk bands, the TSSs show no observable temperature dependence [Figs. 4(a) and 4(b)], with diminished gap in a large temperature range [35]. In $\mathrm{MnBi}_{2} \mathrm{Te}_{4}$, the TSSs can be described by an effective Hamiltonian, $H(\boldsymbol{k})=\sigma_{x} k_{y}-\sigma_{y} k_{x}+\tilde{m}_{z} \sigma_{z}$, where 


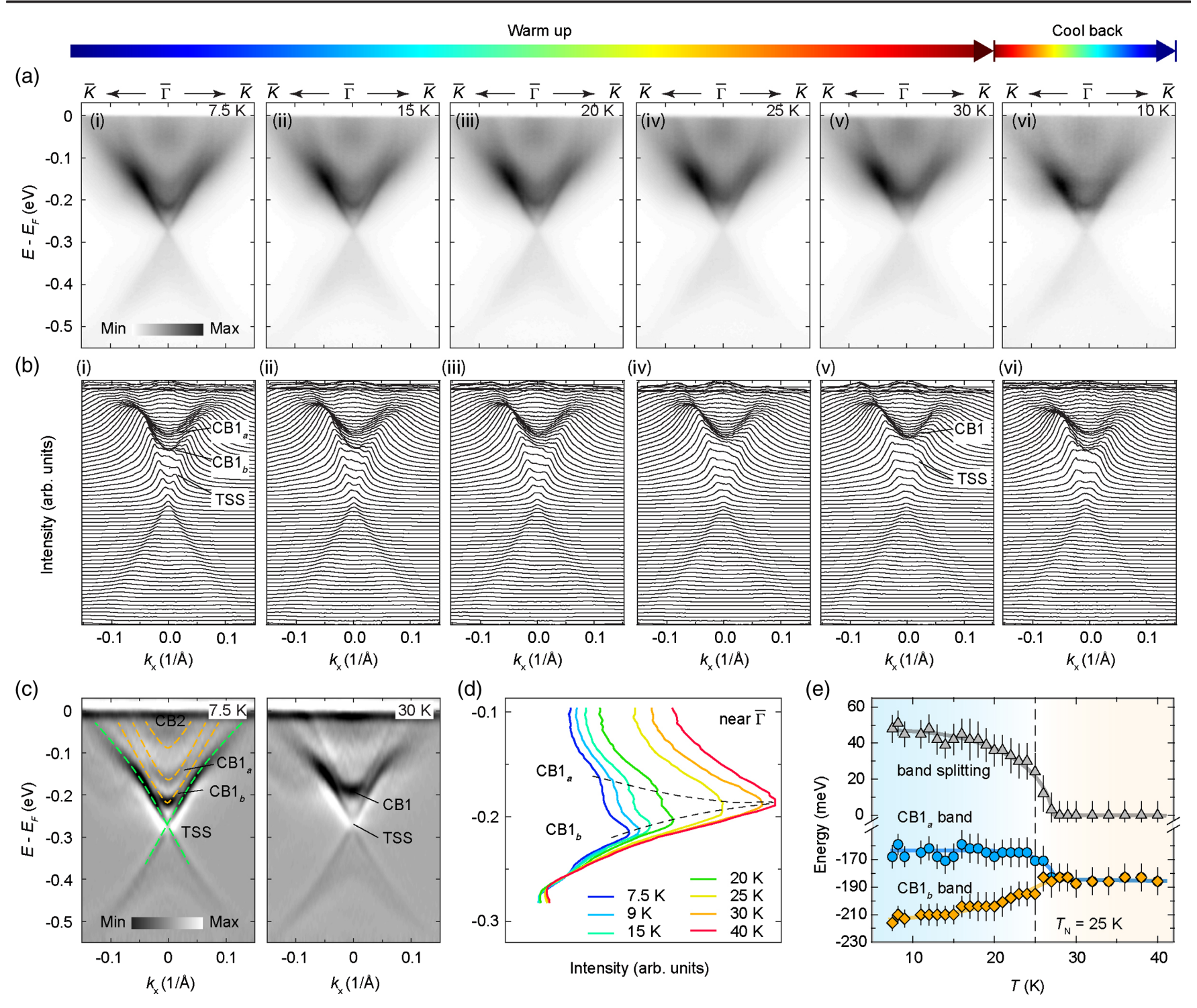

FIG. 4. Temperature evolution of the band structure of $\mathrm{MnBi}_{2} \mathrm{Te}_{4}$. (a),(b) Band dispersions along $\bar{\Gamma} \bar{K}$ (a) and corresponding MDCs (b) at selected temperatures. (c) Side-by-side comparison of the second derivative of ARPES intensity maps at 7.5 and 30 K. (d) Temperature evolution of EDCs at $\bar{\Gamma}$. (e) The energy position of $\mathrm{CB} 1_{a}$ and $\mathrm{CB} 1_{b}$ bands together with the energy difference between them as functions of the temperature showing a band splitting below $27 \mathrm{~K}$. Data were taken with $6.994 \mathrm{eV}$ laser.

$\sigma_{x, y, z}$ are the Pauli matrices for spins and $\tilde{m}_{z}$ is the effective mass induced by exchange interactions on the surface, which could open a surface gap of $2\left|\tilde{m}_{z}\right|$. For $T>T_{N}$, the system becomes paramagnetic, TRS gets preserved, and thus $\mathrm{MnBi}_{2} \mathrm{Te}_{4}$ transits into the time-reversal invariant TI phase, implying a vanishing surface gap. When the temperature is lowered below $T_{N}$, the material will transit into an $A$-type AFM state, as revealed by previous studies [16,17]. Therefore, if the surface magnetism is ideally ordered along the out-of-plane $z$ direction, a surface gap of $\sim 40 \mathrm{meV}$ would develop, as indicated by theoretical calculations [16].

The observed surface gap, however, is vanishingly small, suggesting that the surface magnetism may not be well ordered. The result could presumably be explained by the following mechanisms. Firstly, although the out-of-plane easy axis of the bulk AFM in $\mathrm{MnBi}_{2} \mathrm{Te}_{4}$ has been widely confirmed by various experiments [24,26,39], the interlayer antiferromagnetic interactions could be weaker on the surface than in the bulk, and the magnetic dipole-dipole interactions of the ferromagnetic monolayer typically prefers an in-plane easy axis, which makes the orientation of surface magnetism easily fluctuated. The surface magnetism, once oriented along the in-plane $x$ direction, would contribute an interaction term of $\tilde{m}_{x} \sigma_{x}$ into the effective Hamiltonian, which cannot open the surface gap, as confirmed by recent calculations $[35,40]$. This would shift the Dirac point of TSSs slightly away from the $\bar{\Gamma}$ point. However, the averaged shift over different in-plane orientations would vanish due to the high symmetry of the inplane structure. Secondly, magnetic domains ubiquitously 
exist in AFM materials and cannot be easily eliminated even by field cooling. Since the sign of the exchangeinduced mass depends on the orientation of the surface magnetism, opposing magnetic domains would lead to opposite exchange effects. Indeed, previous x-ray magnetic circular dichroism measurement has shown a signature of the existence of magnetic domains, and its effects on the topological properties of $\mathrm{MnBi}_{2} \mathrm{Te}_{4}$ have been theoretically discussed [24,41]. In particular, it is well known that Diraclike surface states can have unusually large localization length $\lambda_{D}(T)$ caused by the Berry phase of $\pi$ [42] and $\lambda_{D}(T)$ greatly enhances with decreasing temperature. At low temperatures $\lambda_{D}$ is expected to significantly exceed the size of the magnetic domains (typically on the order of $10 \mathrm{~nm}$ ) [43]. Thus, the effective exchange effects experienced by TSSs are contributed by a few magnetic domains, whose averaged effects are weakened by magnetic compensation. From another point of view, gapless chiral boundary modes are topologically protected to exist in the presence of opposing magnetic domains [44]. The gapless modes would play an important role in determining macroscopic properties of TSSs if the domain size is small enough [e.g., compared to $\lambda_{D}(T)$ ]. This could also help to explain why the magnetization-induced surface gap is diminished in $\mathrm{MnBi}_{2} \mathrm{Te}_{4}$.

\section{CONCLUSION}

In conclusion, we have presented a systematic investigation of the electronic structure of the AFM-TI candidate $\mathrm{MnBi}_{2} \mathrm{Te}_{4}$. We observed gapped bulk electronic bands with clear $k_{z}$ dispersion and TSSs with diminished gap as the characteristic topological electronic structure which is attributed to the neutralized net magnetic moment of domains with different magnetization directions. We further observed bulk band splitting possibly related to the surface ferromagnetism caused by the Mn atoms buried in the surface layer. Our results not only reconcile the contradicting results on the origin and magnitude of the previously observed gap in $\mathrm{MnBi}_{2} \mathrm{Te}_{4}$, but also provide important insight into the electronic structure and magnetism of $\mathrm{MnBi}_{2} \mathrm{Te}_{4}$, which is crucial for the understanding of the interplay between magnetism and topology in AFM TIs.

\section{ACKNOWLEDGMENTS}

We thank Jinsong Zhang, Ke He, Zhong Wang, and Yayu Wang for inspiring discussions. This work was supported by the National Natural Science Foundation of China (Grants No. 11774190, No. 11674229, No. 11634009, No. 11774427, and No. 11227902), the National Key R\&D program of China (Grants No. 2017YFA0304600 and No. 2017YFA0305400), EPSRC Platform Grant (Grant No. EP/M020517/1). Y. F. G. acknowledges the support from the Shanghai Pujiang Program (Grant
No. 17PJ1406200). L. X. Y. acknowledges the support from Tsinghua University Initiative Scientific Research Program. Y. W. L. acknowledges the support from China Scholarship Council.

Note added.-Recently, other works reported gapless surface electronic states on $\mathrm{MnBi}_{2} \mathrm{Te}_{4}$ single crystals, which are in good agreement with our observations $[22,45,46]$.

[1] R. Yu, W. Zhang, H.-J. Zhang, S.-C. Zhang, X. Dai, and Z. Fang, Quantized Anomalous Hall Effect in Magnetic Topological Insulators, Science 329, 61 (2010).

[2] C.-Z. Chang, J. Zhang, X. Feng, J. Shen, Z. Zhang, M. Guo, K. Li, Y. Ou, P. Wei et al., Experimental Observation of the Quantum Anomalous Hall Effect in a Magnetic Topological Insulator, Science 340, 167 (2013).

[3] H. Zhang, Y. Xu, J. Wang, K. Chang, and S.-C. Zhang, Quantum Spin Hall and Quantum Anomalous Hall States Realized in Junction Quantum Wells, Phys. Rev. Lett. 112, 216803 (2014).

[4] C.-Z. Chang, W. Zhao, D. Y. Kim, H. Zhang, B. A. Assaf, D. Heiman, S.-C. Zhang, C. Liu, M. H. W. Chan, and J.S. Moodera, High-Precision Realization of Robust Quantum Anomalous Hall State in a Hard Ferromagnetic Topological Insulator, Nat. Mater. 14, 473 (2015).

[5] D. Xiao, J. Jiang, J.-H. Shin, W. Wang, F. Wang, Y.-F. Zhao, C. Liu, W. Wu, M. H. W. Chan et al., Realization of the Axion Insulator State in Quantum Anomalous Hall Sandwich Heterostructures, Phys. Rev. Lett. 120, 056801 (2018).

[6] Y. Hou and R. Wu, Axion Insulator State in a Ferromagnet/ Topological Insulator/Antiferromagnet Heterostructure, Nano Lett. 19, 2472 (2019).

[7] M. Mogi, M. Kawamura, R. Yoshimi, A. Tsukazaki, Y. Kozuka, N. Shirakawa, K. S. Takahashi, M. Kawasaki, and Y. Tokura, A Magnetic Heterostructure of Topological Insulators as a Candidate for an Axion Insulator, Nat. Mater. 16, 516 (2017).

[8] P. Tang, Q. Zhou, G. Xu, and S.-C. Zhang, Dirac Fermions in an Antiferromagnetic Semimetal, Nat. Phys. 12, 1100 (2016).

[9] J. Wang, Magnetic Dirac Semimetals in Three Dimensions, arXiv:1701.00896.

[10] X. Wan, A. M. Turner, A. Vishwanath, and S. Y. Savrasov, Topological Semimetal and Fermi-Arc Surface States in the Electronic Structure of Pyrochlore Iridates, Phys. Rev. B 83, 205101 (2011).

[11] G. Xu, H. Weng, Z. Wang, X. Dai, and Z. Fang, Chern Semimetal and the Quantized Anomalous Hall Effect in $\mathrm{HgCr}_{2} \mathrm{Se}_{4}$, Phys. Rev. Lett. 107, 186806 (2011).

[12] E. Liu, Y. Sun, N. Kumar, L. Muechler, A. Sun, L. Jiao, S.-Y. Yang, D. Liu, A. Liang et al., Giant Anomalous Hall Effect in a Ferromagnetic Kagome-Lattice Semimetal, Nat. Phys. 14, 1125 (2018).

[13] Q. Wang, Y. Xu, R. Lou, Z. Liu, M. Li, Y. Huang, D. Shen, H. Weng, S. Wang, and H. Lei, Large Intrinsic Anomalous Hall Effect in Half-Metallic Ferromagnet $\mathrm{Co}_{3} \mathrm{Sn}_{2} \mathrm{~S}_{2}$ with Magnetic Weyl Fermions, Nat. Commun. 9, 3681 (2018). 
[14] Q. L. He, L. Pan, A. L. Stern, E. C. Burks, X. Che, G. Yin, J. Wang, B. Lian, Q. Zhou et al., Chiral Majorana Fermion Modes in a Quantum Anomalous Hall InsulatorSuperconductor Structure, Science 357, 294 (2017).

[15] R.S. K. Mong, A. M. Essin, and J. E. Moore, Antiferromagnetic Topological Insulators, Phys. Rev. B 81, 245209 (2010).

[16] J. Li, Y. Li, S. Du, Z. Wang, B.-L. Gu, S.-C. Zhang, K. He, W. Duan, and Y. Xu, Intrinsic Magnetic Topological Insulators in van der Waals Layered $\mathrm{MnBi}_{2} \mathrm{Te}_{4}$-Family Materials, Sci. Adv. 5, eaaw5685 (2019).

[17] D. Zhang, M. Shi, T. Zhu, D. Xing, H. Zhang, and J. Wang, Topological Axion States in the Magnetic Insulator $\mathrm{MnBi}_{2} \mathrm{Te}_{4}$ with the Quantized Magnetoelectric Effect, Phys. Rev. Lett. 122, 206401 (2019).

[18] M. M. Otrokov, I. P. Rusinov, M. Blanco-Rey, M. Hoffmann, A. Y. Vyazovskaya, S. V. Eremeev, A. Ernst, P. M. Echenique, A. Arnau, and E. V. Chulkov, Unique Thickness-Dependent Properties of the van der Waals Interlayer Antiferromagnet $\mathrm{MnBi}_{2} \mathrm{Te}_{4}$ Films, Phys. Rev. Lett. 122, 107202 (2019).

[19] S. Zhang, R. Wang, X. Wang, B. Wei, H. Wang, G. Shi, F. Wang, B. Jia, Y. Ouyang et al., Experimental Observation of the Gate-Controlled Reversal of the Anomalous Hall Effect in the Intrinsic Magnetic Topological Insulator $\mathrm{MnBi}_{2} \mathrm{Te}_{4}$ Device, arXiv:1905.04839.

[20] Y. Deng, Y. Yu, M. Z. Shi, J. Wang, X. H. Chen, and Y. Zhang, Magnetic-Field-Induced Quantized Anomalous Hall Effect in Intrinsic Magnetic Topological Insulator $\mathrm{MnBi}_{2} \mathrm{Te}_{4}$, arXiv:1904.11468.

[21] C. Liu, Y. Wang, H. Li, Y. Wu, Y. Li, J. Li, K. He, Y. Xu, J. Zhang et al., Quantum Phase Transition from Axion Insulator to Chern Insulator in $\mathrm{MnBi}_{2} \mathrm{Te}_{4}$, arXiv:1905 .00715 .

[22] B. Chen, F. Fei, D. Zhang, B. Zhang, W. Liu, S. Zhang, P. Wang, B. Wei, Y. Zhang et al., Intrinsic Magnetic Topological Insulator Phases in the $\mathrm{Sb}$ Doped $\mathrm{MnBi}_{2} \mathrm{Te}_{4}$ Bulks and Thin Flakes, Nat. Commun. 10, 4469 (2019).

[23] S. H. Lee, Y. Zhu, Y. Wang, L. Miao, T. Pillsbury, H. Yi, S. Kempinger, J. Hu, C. A. Heikes, P. Quarterman et al., Spin Scattering and Noncollinear Spin Structure-Induced Intrinsic Anomalous Hall Effect in Antiferromagnetic Topological Insulator $\mathrm{MnBi}_{2} \mathrm{Te}_{4}$, Phys. Rev. Res. 1, 012011 (2019).

[24] M. M. Otrokov, I. I. Klimovskikh, H. Bentmann, A. Zeugner, Z. S. Aliev, S. Gass, A. U. B. Wolter, A. V. Koroleva, D. Estyunin et al., Prediction and Observation of the First Antiferromagnetic Topological Insulator, arXiv: 1809.07389.

[25] R. C. Vidal, H. Bentmann, T. R. F. Peixoto, A. Zeugner, S. Moser, C. H. Min, S. Schatz, K. Kißner, M. Ünzelmann, C. I. Fornari et al., Surface States and Rashba-Type Spin Polarization in Antiferromagnetic $\mathrm{MnBi}_{2} \mathrm{Te}_{4}(0001)$, Phys. Rev. B 100, 121104(R) (2019).

[26] A. Zeugner, F. Nietschke, A. U. B. Wolter, S. Gaß, R. C. Vidal, T. R. F. Peixoto, D. Pohl, C. Damm, A. Lubk et al., Chemical Aspects of the Candidate Antiferromagnetic Topological Insulator $\mathrm{MnBi}_{2} \mathrm{Te}_{4}$, Chem. Mater. 31, 2795 (2019).
[27] Y. Gong, J. Guo, J. Li, K. Zhu, M. Liao, X. Liu, Q. Zhang, L. Gu, L. Tang et al., Experimental Realization of an Intrinsic Magnetic Topological Insulator, Chin. Phys. Lett. 36, 076801 (2019).

[28] H. Fu, C.-X. Liu, and B. Yan, Exchange Bias and Quantum Anomalous Hall Effect in the $\mathrm{MnBi}_{2} \mathrm{Te}_{4}-\mathrm{CrI}_{3}$ Heterostructure, arXiv:1908.04322.

[29] H. Li, S. Liu, C. Liu, J. Zhang, Y. Xu, R. Yu, Y. Wu, Y. Zhang, and S. Fan, Antiferromagnetic Topological Insulator $\mathrm{MnBi}_{2} \mathrm{Te}_{4}$ : Synthesis and Magnetic Properties, arXiv:1907 13018

[30] J. P. Perdew, K. Burke, and M. Ernzerhof, Generalized Gradient Approximation Made Simple, Phys. Rev. Lett. 77, 3865 (1996).

[31] Q. Wu, S. Zhang, H.-F. Song, M. Troyer, and A. A. Soluyanov, WannierTools: An Open-Source Software Package for Novel Topological Materials, Comput. Phys. Commun. 224, 405 (2018).

[32] A. A. Mostofi, J. R. Yates, G. Pizzi, Y.-S. Lee, I. Souza, D. Vanderbilt, and N. Marzari, An Updated Version of wannier90: A Tool for Obtaining Maximally-Localised Wannier Functions, Comput. Phys. Commun. 185, 2309 (2014).

[33] D. S. Lee, T.-H. Kim, C.-H. Park, C.-Y. Chung, Y. S. Lim, W.-S. Seo, and H.-H. Park, Crystal Structure, Properties and Nanostructuring of a New Layered Chalcogenide Semiconductor, $\mathrm{Bi}_{2} \mathrm{MnTe}_{4}$, CrystEngComm 15, 5532 (2013).

[34] J. Q. Yan, Q. Zhang, T. Heitmann, Z. Huang, K. Y. Chen, J. G. Cheng, W. Wu, D. Vaknin, B. C. Sales, and R. J. McQueeney, Crystal Growth and Magnetic Structure of $\mathrm{MnBi}_{2} \mathrm{Te}_{4}$, Phys. Rev. Mater. 3, 064202 (2019).

[35] See Supplemental Material at http://link.aps.org/ supplemental/10.1103/PhysRevX.9.041040 for (I) sample characterization, (II) $a b$ initio calculation of topological surface states, (III) additional photon-energy-dependent measurements, (IV) additional temperature-dependent measurements, (V) topological surface state with diminished gap measured at $82 \mathrm{~K}$, (VI) extraction of bulk band gap, (VII) Dirac cone simulation, and (VIII) analysis of EDC line shape around the Dirac point.

[36] Y. L. Chen, J. G. Analytis, J.-H. Chu, Z. K. Liu, S.-K. Mo, X. L. Qi, H. J. Zhang, D. H. Lu, X. Dai et al., Experimental Realization of a Three-Dimensional Topological Insulator, $\mathrm{Bi}_{2} \mathrm{Te}_{3}$, Science 325, 178 (2009).

[37] Y. L. Chen, Z. K. Liu, J. G. Analytis, J. H. Chu, H. J. Zhang, B. H. Yan, S. K. Mo, R. G. Moore, D. H. Lu et al., Single Dirac Cone Topological Surface State and Unusual Thermoelectric Property of Compounds from a New Topological Insulator Family, Phys. Rev. Lett. 105, 266401 (2010).

[38] A. Chikina, M. Höppner, S. Seiro, K. Kummer, S. Danzenbächer, S. Patil, A. Generalov, M. Güttler, Y. Kucherenko et al., Strong Ferromagnetism at the Surface of an Antiferromagnet Caused by Buried Magnetic Moments, Nat. Commun. 5, 3171 (2014).

[39] J. Cui, M. Shi, H. Wang, F. Yu, T. Wu, X. Luo, J. Ying, and X. Chen, Transport Properties of Thin Flakes of the Antiferromagnetic Topological Insulator $\mathrm{MnBi}_{2} \mathrm{Te}_{4}$, Phys. Rev. B 99, 155125 (2019). 
[40] J. Li, C. Wang, Z. Zhang, B.-L. Gu, W. Duan, and Y. Xu, Magnetically Controllable Topological Quantum Phase Transitions in the Antiferromagnetic Topological Insulator $\mathrm{MnBi}_{2} \mathrm{Te}_{4}$, Phys. Rev. B 100, 121103(R) (2019).

[41] J. Zhang, Z. Liu, and J. Wang, In-Plane Magnetic-FieldInduced Quantum Anomalous Hall Plateau Transition, Phys. Rev. B 100, 165117 (2019).

[42] K. Nomura, M. Koshino, and S. Ryu, Topological Delocalization of Two-Dimensional Massless Dirac Fermions, Phys. Rev. Lett. 99, 146806 (2007).

[43] K. Yasuda, M. Mogi, R. Yoshimi, A. Tsukazaki, K. S. Takahashi, M. Kawasaki, F. Kagawa, and Y. Tokura, Quantized Chiral Edge Conduction on Domain Walls of a Magnetic Topological Insulator, Science 358, 1311 (2017).

[44] X.-L. Qi and S.-C. Zhang, Topological Insulators and Superconductors, Rev. Mod. Phys. 83, 1057 (2011).

[45] Y.-J. Hao, P. Liu, Y. Feng, X.-M. Ma, E. F. Schwier, M. Arita, $\mathrm{S}$. Kumar, C. Hu, R. Lu, M. Zeng et al., this issue, Gapless Surface Dirac Cone in Antiferromagnetic Topological Insulator $\mathrm{MnBi}_{2} \mathrm{Te}_{4}$, Phys. Rev. X 9, 041038 (2019).

[46] H. Li, S.-Y. Gao, S.-F. Duan, Y.-F. Xu, K.-J. Zhu, S.-J. Tian, W.-H. Fan, Z.-C. Rao, J.-R. Huang et al., preceding paper, Dirac Surface States in Intrinsic Magnetic Topological Insulators $\mathrm{EuSn}_{2} \mathrm{As}_{2}$ and $\mathrm{MnBi}_{2} \mathrm{Te}_{4}$, Phys. Rev. X 9 , 041039 (2019). 